\title{
Does the deficiency of Aire in mice really resemble human APECED?
}

\section{Eliisa Kekäläinen, Aaro Miettinen and T. Petteri Arstila}

In a recent Perspective $\operatorname{article}^{1}$ Mathis and Benoist describe an impressive decade of autoimmune polyendocrinopathy-candidiasis-ectodermal dystrophy (APECED) research since the discovery of the mutated autoimmune regulator (AIRE) gene as the underlying cause of this rare inherited autoimmune disease.

The elucidation of the role of Aire in the regulation of ectopic gene expression in the mouse thymus is indeed a major advance ${ }^{2,3}$. However, the authors also assert that major arguments suggesting differences in AIRE in humans and mice have been invalidated. From a translational point of view this is a crucial point, and, as researchers studying both AIRE-deficient mice and humans, we beg to differ.

The clinical difference between patients with APECED ${ }^{4}$ and the originally described Aire-knockout mouse ${ }^{2,5}$, which was on a $\mathrm{C} 57 \mathrm{Bl} / 6 \times 129 / \mathrm{Cv}$ genetic background, is acknowledged by Mathis and Benoist, but they suggest that because on other genetic backgrounds the mouse disease is very severe, this difference is irrelevant. They do not mention that in a paper they cite ${ }^{6}$ Aire-deficient non-obese diabetic (NOD) mice are in fact the only animals to become sick.

In the NOD background the disease manifests as fulminant exocrine pancreatitis, a characteristic which has not been described in human APECED ${ }^{4}$. On the other hand, diabetes is often part of APECED, but, in Aire-deficient NOD mice, pancreatic islets are intact even though insulin expression in the mouse thymus is controlled by Aire ${ }^{2}$. As for the other mouse strains, Mathis and Benoist inaccurately equate immunopathological findings, such as autoantibodies and infiltrating lymphocytes, with autoimmune disease, without any evidence of excess mortality, morbidity or even tissue damage. Analogous situations are commonplace in medicine. It is well known that a certain fraction of apparently healthy individuals will test positive for autoantibodies $^{7,8}$. Yet there is no evidence of any autoimmune disease, and in the majority of these individuals the presence of autoantibodies does not predict future autoimmunity. Similar to the situation in Aire-knockout mice, these individuals have immunopathological findings but no disease. By contrast, without appropriate hormone replacement therapy the main endocrinological deficiencies in APECED are fatal.

A further claim in the article is that differences in autoantigens ${ }^{9}$ and target organs are only to be expected, given the differences in the genes that encode MHC molecules in the two species. This disregards the fact that, in patients with APECED, the targets of autoimmunity are often quite conserved between individuals ${ }^{10}$, and the phenotype of the disease is only weakly correlated with HLA type or other non-AIRE genes $^{11-13}$. Indeed, the strong influence of non-Aire genes on the mouse phenotype is another clear difference between the two species, not a reason to dismiss the discrepancies.

We believe that, far from invalidating, the current data tend to support the view that AIRE-deficiency leads to a different phenotype in humans and mice. This may be due to differences in AIRE function or, perhaps more probably, in the physiological environment in which AIRE function takes place. We have elsewhere ${ }^{14}$ suggested that thymic architecture and the role of Hassall's corpuscles in supporting human regulatory T-cell development ${ }^{15}$ might be involved in the dissimilar clinical outcome in the two species. Uncovering the reasons for the species-dependent differences should reveal important aspects of human autoimmunity.

\section{Eliisa Kekäläinen and T. Petteri Arstila are at the Haartman Institute, Department of Immunology, University of Helsinki, Finland. \\ Aaro Miettinen is at HUSLAB, Helsinki University Hospital, Helsinki, Finland. \\ Correspondence to T. P. A. e-mail:petteri.arstila@helsinki.fi doi: 10.1038/nri2136-c1}

1. Mathis, D. \& Benoist, C. A decade of AIRE. Nature Rev. Immunol. 7, 645-650 (2007).

2. Anderson, M. S. et al. Projection of an immunological self shadow within the thymus by the aire protein. Science 298, 1395-1401 (2002).

3. Liston, A., Lesage, S., Wilson, J., Peltonen, L. \& Goodnow, C. C. Aire regulates negative selection of organ-specific T cells. Nature Immunol. 4, 350-354 (2003).

4. Perheentupa, J. Autoimmune polyendocrinopathy-candidiasis-ectodermal dystrophy. J. Clin. Endocrinol. Metab. 91, 2843-2850 (2006).

5. Ramsey, C. et al. Aire deficient mice develop multiple features of APECED phenotype and show altered immune response. Hum. Mol. Genet. 11, 397-409 (2002).

6. Jiang, W., Anderson, M. S., Bronson, R., Mathis, D. \& Benoist, C. Modifier loci condition autoimmunity provoked by Aire deficiency. J. Exp. Med. 202, 805-815 (2005)

7. Lacroix-Desmazes, S. et al. Self-reactive antibodies (natural autoantibodies) in healthy individuals. J. Immunol. Methods 216, 117-137 (1998).

8. Pisetsky, D. S. Anti-DNA and autoantibodies. Curr. Opin. Rheumatol. 12, 364-368 (2000).

9. Pöntynen, N. et al. Aire deficient mice do not develop the same profile of tissue-specific autoantibodies as APECED patients. J. Autoimmun. 27, 96-104 (2006)

10. Soderbergh, A. et al. Prevalence and clinical associations of 10 defined autoantibodies in autoimmune polyendocrine syndrome type I. J. Clin. Endocrinol. Metab. 89, 557-562 (2004).

11. Gylling, M. et al. ss-cell autoantibodies, human leukocyte antigen II alleles, and type 1 diabetes in autoimmune

polyendocrinopathy-candidiasis-ectodermal dystrophy. J. Clin. Endocrinol. Metab. 85, 4434-4440 (2000).

12. Halonen, M. et al. AIRE mutations and human leukocyte antigen genotypes as determinants of the autoimmune

polyendocrinopathy-candidiasis-ectodermal dystrophy phenotype. J. Clin. Endocrinol. Metab. 87, 25682574 (2002).

13. Gylling, M. et al. The hypoparathyroidism of autoimmune

polyendocrinopathy-candidiasis-ectodermal dystrophy protective effect of male sex. J. Clin. Endocrinol. Metab. 88, 4602-4608 (2003).

14. Kekäläinen, E. et al. A defect of regulatory T cells in patients with autoimmune polyendocrinopathy-candidiasis-ectodermal dystrophy. J. Immunol. 178, 1208-1215 (2007).

15. Watanabe, N. et al. Hassall's corpuscles instruct dendritic cells to induce $\mathrm{CD} 4{ }^{+} \mathrm{CD} 25^{+}$regulatory $\mathrm{T}$ cells in human thymus. Nature 436, 1181-1185 (2005). 